\title{
Amygdalo-Hypothalamic Circuit Allows Learned Cues to Override Satiety and Promote Eating
}

\author{
Gorica D. Petrovich, Barry Setlow, Peter C. Holland, and Michela Gallagher \\ Department of Psychological and Brain Sciences, Johns Hopkins University, Baltimore, Maryland 21218
}

Organisms eat not only in a response to signals related to energy balance. Eating also occurs in response to "extrinsic," or environmental, signals, including learned cues. Such cues can modify feeding based on motivational value acquired through association with either rewarding or aversive events. We provide evidence that a specific brain system, involving connections between basolateral amygdala and the lateral hypothalamus, is crucial for allowing learned cues (signals that were paired with food delivery when the animal was hungry) to override satiety and promote eating in sated rats. In an assess- ment of second-order conditioning, we also found that disconnection of this circuitry had no effect on the ability of a conditioned cue to support new learning. Knowledge about neural systems through which food-associated cues specifically control feeding behavior provides a defined model for the study of learning that may be informative for understanding mechanisms that contribute to eating disorders and more moderate forms of overeating.

Key words: amygdala; hypothalamus; eating; feeding behavior; learning; goal-directed behavior; motivation
Mild to severe obesity, estimated to affect $\sim 60 \%$ of the adult population in developed countries, is a risk factor for a range of diseases (United States Department of Health and Human Services, 1999). Despite adverse health consequences, difficulties in achieving and maintaining weight control are common. Overeating is attributable, at least in part, to the fact that food consumption is powerfully influenced by a variety of environmental factors that are unrelated to energy requirements (Rodin, 1981; Booth, 1989). Eating can be socially facilitated (De Castro, 1997), and cues that become associated with food when hunger prevails can increase eating in satiated states (Weingarten, 1983).

Recent research is beginning to define the neural systems through which such psychological processes influence eating. Under conditions that strongly potentiate feeding, laboratory rats with neurotoxic lesions of the basolateral amygdalar area (BLA) [including basolateral ("basal"), basomedial ("accessory basal"), and lateral nuclei] fail to increase eating in the presence of a conditioned stimulus (CS) that was previously paired with food (Gallagher, 2000; Holland et al., 2002). The BLA has anatomical connections with neural circuits in the hypothalamus that control feeding behavior (Elmquist et al., 1999; Swanson, 2000; DeFalco et al., 2001; Petrovich et al., 2001). It sends substantial, topographically organized projections to the lateral hypothalamic area (LHA) (Petrovich et al., 2001), which forms part of the feeding circuit and historically has been linked to initiation of feeding (Elmquist et al., 1999).

The current investigation examined potentiation of feeding by a CS in rats with a preparation that disconnects the BLA and the LHA. Here we report that the BLA-LHA system is crucial for allowing learned cues to override satiety signals and stimulate

Received May 20, 2002; revised July 8, 2002; accepted July 15, 2002.

This work was supported by grants from the National Institutes of Health. We thank Summer Nugent for excellent technical support and Dr. Jennifer Bizon for comments on a previous version of this manuscript.

Correspondence should be addressed to Michela Gallagher, Department of Psychological and Brain Sciences, Johns Hopkins University, 3400 North Charles Street, Baltimore, MD 21218. E-mail: michela@jhu.edu.

Copyright (C) 2002 Society for Neuroscience $0270-6474 / 02 / 228748-06 \$ 15.00 / 0$ eating in sated states. We further show that the BLA-LHA system is specifically important for the control of eating by learned signals, because it does not regulate baseline eating or the rate at which rats gain weight when fed ad libitum.

\section{MATERIALS AND METHODS}

Subjects. Experimentally naive, male Long-Evans rats (Charles River Laboratories, Raleigh, NC), weighing $\sim 300 \mathrm{gm}$ on arrival in the vivarium, were individually caged, maintained on a $12 \mathrm{hr}$ light/dark cycle, and given ad libitum access to food and water, except as otherwise noted. After 1 week acclimation to vivarium conditions, during which time they were handled extensively, rats were given contralateral, ipsilateral, or sham-contralateral lesions of BLA and LHA and then allowed to recover for 1 week before undergoing behavioral procedures.

Surgical methods. All surgeries were performed under aseptic conditions using isoflurane gas for induction and maintenance of anesthesia, using a stereotaxic frame (Kopf Instruments, Tujunga, CA). Neurotoxic unilateral lesions were made with NMDA (Sigma, St. Louis, MO) in a $0.1 \mathrm{M}$ phosphate buffer solution, using a concentration of $12.5 \mathrm{mg} / \mathrm{ml}$ for BLA and $20 \mathrm{mg} / \mathrm{ml}$ for LHA lesions. The placement of lesions was balanced, such that there were approximately equal numbers of rats with lesions in the left or right hemispheres in each group. For BLA, a total of $0.3 \mu \mathrm{l}$ was infused [the flat skull coordinates from bregma were as follows: anteroposterior (AP), $-2.70 \mathrm{~mm}$; mediolateral (ML), \pm 4.80 $\mathrm{mm}$; and dorsoventral (DV), -8.70 and $-8.40 \mathrm{~mm} ; 0.2 \mu \mathrm{l}$ was infused at the deeper site and $0.1 \mu \mathrm{l}$ at the shallow site). For LHA, a total of 0.35 $\mu \mathrm{l}$ was infused (the flat skull coordinates from bregma were as follows: AP, $-2.70 \mathrm{~mm}$; ML, $\pm 1.40 \mathrm{~mm}$; and DV,$-8.90 \mathrm{~mm}$ ). Sham lesions of both structures were made by infusing phosphate buffer vehicle alone. Infusions were made through a 30 gauge needle attached by a length of plastic tubing to a $10 \mu \mathrm{l}$ microsyringe (Hamilton, Reno, NV) mounted on a syringe pump (Sage Instruments, Boston, MA). After each infusion, the needle was left in place for $4 \mathrm{~min}$ to allow for diff usion. Immediately after surgery, rats were given diazepam (Sigma) in a $5 \mathrm{mg} / \mathrm{ml}$ solution (mixture of $80 \%$ propylene glycol and $20 \%$ ethanol) at a dose of $5 \mathrm{mg} / \mathrm{kg}$ to prevent seizures.

Apparatus. Six identical behavioral chambers $(30 \times 24 \times 30 \mathrm{~cm}$; Colbourn Instruments, Allentown, PA), each with a grid floor, aluminum top and sides, and a transparent Plexiglas back and front, were used for training and testing. The interior length of each chamber was reduced by positioning a transparent Plexiglas partition at an angle such that the length of the floor was reduced to $22 \mathrm{~cm}$, but the length at the top of the box remained unaltered. On the side wall opposite the Plexiglas partition, each chamber contained a recessed food cup $(3.2 \times 4.2 \mathrm{~cm})$, into which 
food pellets (45 mg; P. J. Noyes, Lancaster, NH) were delivered. Dim background illumination was provided by two $25 \mathrm{~W}$ red bulbs, each placed $\sim 1.5 \mathrm{~m}$ from the test chambers. Masking noise $(60 \mathrm{~dB})$ was provided by ventilation fans located outside each box. A 10 sec tone (1500 $\mathrm{Hz}, 70 \mathrm{~dB})$ and a $10 \mathrm{sec}$ white noise $(70 \mathrm{~dB})$ were used as CS + and CS- . For approximately half of the animals in each group, the tone served as the $\mathrm{CS}+$, whereas the white noise served as the $\mathrm{CS}+$ for the remaining half. The unconditioned stimulus consisted of two $45 \mathrm{mg}$ food pellets (P. J. Noyes) delivered at the termination of the CS+. A $4 \mathrm{~W}$ light mounted on the box wall $20 \mathrm{~cm}$ above the food cup was used for the light CS2 in second-order conditioning.

Video cameras attached to videocassette recorders were placed $1 \mathrm{~m}$ in front of the test chambers to record behavior for a $10 \mathrm{sec}$ period before and during stimulus presentation. Stimulus presentation and videocassette recorders were controlled by LabView (National Instruments) software run on Macintosh computers (Apple Computers, Cupertino, CA).

Behavioral procedures. Before behavior training, rats were gradually reduced to $85 \%$ of their ad libitum weights. After a shaping procedure (Setlow et al., 2002) in which rats learned to eat from the food cup, rats received two sessions (one session per day) of CS-food pairing. Each 32 min session consisted of eight presentations of the $10 \mathrm{sec}$ CS + (tone or noise), immediately followed by delivery of two pellets into the food cup. In each of the next eleven 32 min discrimination training sessions, the rats received two reinforced presentations of the CS+ intermixed with six nonreinforced presentations of the other auditory stimulus $(\mathrm{CS}-$ ).

After completion of first-order conditioning, rats were given $9 \mathrm{~d}$ of $a d$ libitum access to food in their home cage. On the last two of these days, the rats received consumption tests (one per day) in the test chambers: one with the $\mathrm{CS}+$ and the other with $\mathrm{CS}-$. The protocol on each test day began with ad libitum access to food pellets in the home cage for $1 \mathrm{hr}$, immediately followed by a $10 \mathrm{~min}$ pretest session and a 10 min test session in the behavioral chambers. The 10 min pretest was included to provide a "baseline" for food consumption in the behavioral chamber and to reduce context-dependent consumption during the test so that potentiation of eating by the CS + would be readily apparent. For the pretest, rats were placed into the experimental chambers with 50 food pellets available in the food cup. At the completion of the pretest, rats were removed from the chambers, pellets remaining in the food cup were collected for counting, and food cups were refilled with 50 new pellets. Rats were then promptly returned to the chambers for the 10 min test. During the test, ten $10 \mathrm{sec} \mathrm{CS}+$ or CS - were presented. Both the order of the tests (CS+ or CS - on the first day) and identity of the CS+ (tone or noise) were counterbalanced. At the end of the test, rats were removed, and pellets remaining in the food cup were counted.

After the completion of tests for CS potentiation of feeding, all rats were again gradually reduced to $85 \%$ of their current ad libitum weights and then received two "reminder" sessions of $\mathrm{CS}+-$ food pairings (identical to the first two training sessions; see above). For second-order conditioning, each of the original groups (sham, ipsilateral, and contralateral) was subdivided into a "paired" group, which received presentations of the $10 \mathrm{sec}$ light CS2 paired with (immediately followed by) the first-order $\mathrm{CS}+$, and an "unpaired" group, which received the same number of light and CS + presentations that were explicitly unpaired. The unpaired groups were included to control for non-associative increases in responding to the light CS2. For both paired and unpaired groups, the first half of session 1 began with a CS +- food reminder trial, followed by three presentations of the light alone to evaluate unconditioned responding to the light. In the second half of session 1 , as well as in each half of sessions 2-8, the paired groups received three trials of the light CS2 paired with the auditory CS+; the unpaired groups received three trials each of auditory CS + and light CS2 unpaired. All groups also received one $\mathrm{CS}+-$ food reminder trials in each half session.

Behavioral observations. Observations were made from the videotapes of the behavioral conditioning sessions by experimenters who were "blind" to group assignments. The observations were paced by auditory signals (at $1.25 \mathrm{sec}$ intervals) recorded onto the tapes. At each observation, only one behavior was recorded. The primary measure of conditioning [conditioned responses (CRs)] to the auditory (in the first-order discrimination phase) and visual (in the second-order conditioning phase) CSs, was the percentage of time the rats spent expressing food cup behavior during the CS intervals. Food cup behavior consists of nose pokes into the recessed food cup, standing motionless in front of the food cup, or short, rapid, horizontal, or vertical head jerks (in the vicinity of the food cup).
Statistics. Behavioral data were analyzed using nonparametric statistics (Kruskall-Wallis, Mann-Whitney, and Wilcoxon signed-rank tests when appropriate). In all cases, $p<0.05$ was considered significant.

Histological procedure. After completion of all behavioral procedures, rats were given an overdose of pentobarbital $(100 \mathrm{mg} / \mathrm{kg})$ and perfused intracardially with $0.9 \%$ saline, followed by $4 \%$ Formalin in $0.1 \mathrm{~m}$ PBS. The brains were removed and stored in the Formalin solution used for perfusion for $48-72 \mathrm{hr}$ and then transferred to $12 \%$ sucrose in $0.1 \mathrm{M}$ PBS for $24 \mathrm{hr}$. The brains were sliced on a freezing microtome, and coronal sections $(40 \mu \mathrm{m})$ collected through the areas of the BLA and LHA were mounted on slides and Nissl stained. Lesion placements were verified under a light microscope and drawn onto plates adapted from the atlas of Swanson (1992).

\section{RESULTS \\ Histology}

The BLA-LHA system was disconnected by making asymmetrical lesions (unilateral lesions of BLA and LHA on opposite sides of the brain), using the neurotoxin NMDA (Fig. 1A,B). Because BLA outputs are predominantly ipsilateral (McDonald and Culberson, 1986; Swanson and Petrovich, 1998), this preparation disconnected the BLA and LHA in both hemispheres (contralateral group, $n=14$ animals with acceptable lesions) without disturbing other functional circuits involving each of these structures. A control group of rats (ipsilateral group, $n=12$ animals with acceptable lesions) had an equivalent amount of damage with unilateral lesions of BLA and LHA placed on the same side of the brain, sparing the BLA-LHA system in one hemisphere. Rats with contralaterally placed sham lesions $(n=15)$ of BLA and LHA were included to control for any effects attributable to surgical procedures alone.

Lesions were rejected if there was $<50 \%$ damage to BLA or LHA or if there was more than minimal $(>10 \%)$ damage to the adjoining regions (the central nucleus and cortical regions for the BLA, and medial hypothalamus and thalamus for the LHA). In most cases, the BLA lesions were confined specifically to the basolateral nucleus (also referred to as basal nucleus), with some additional damage to the lateral and basomedial (accessory basal) nuclei in the case of the largest lesions (Fig. $1 A$ ). The median extent of damage for acceptable lesions was $\sim 70 \%$ in the BLA and $\sim 75 \%$ in the LHA. Within the BLA, in all acceptable lesions, there was $>90 \%$ damage to the basolateral nucleus specifically. There were no differences in size or location between the ipsilateral and contralateral lesions of the BLA or LHA.

\section{Behavior: CS potentiation}

The disconnection of the BLA-LHA system did not affect auditory Pavlovian discrimination learning (first-order conditioning). All groups of rats acquired discrimination rapidly as shown in Figure 2. CRs directed to the food cup during the occurrence of an auditory CS that predicted food delivery (CS+) were significantly elevated compared with CRs during another auditory stimulus that was not paired with food $(\mathrm{CS}-$ ) for all groups (Wilcoxon signed rank; sham group, $p=0.0022$; ipsilateral group, $p=$ 0.0077 ; contralateral group, $p=0.0033$ ). No significant effects were evident in a Kruskall-Wallis analysis comparing the CRs with $\mathrm{CS}+\left(H_{(2)}=2.567 ; p=0.2770\right)$ or CRs with CS $-\left(H_{(2)}=\right.$ $0.622 ; p=0.7328)$ among groups with different lesion treatments (sham, ipsilateral, or contralateral).

After Pavlovian auditory discrimination training, which was conducted in a food-restricted state, rats were allowed food ad libitum for 1 week. Consumption tests for potentiated feeding were then performed in the sated condition on 2 consecutive days, when food was available in the test apparatus in the presence of 
$\mathbf{A}$

Figure 1. Histology. A, The extent of the largest (enclosed black area) and smallest (filled black area) acceptable lesions at several rostrocaudal levels for all rats in the contralateral and ipsilateral groups. Except for minor mechanical damage along the injector tracks, no damage was evident in any of the sham-lesioned brains. Plates adapted from the atlas of Swanson (1992). B, Representative photomicrographs of lesion and sham histology. Arrows denote lesion borders. Scale bar, $0.5 \mathrm{~mm}$. Amygdala: $B L A$, Basolateral area (includes $B L, B M A$, and $L A$ ); $B L$, basolateral (basal); $B M A$, basomedial (accessory basal); $C E A$, central; $L A$, lateral nuclei. Hypothalamus: $D M H$, Dorsomedial nucleus; $f x$, fornix; $L H A$, lateral area; $m t t$, mammillothalamic tract; $V M H$, ventromedial nucleus.
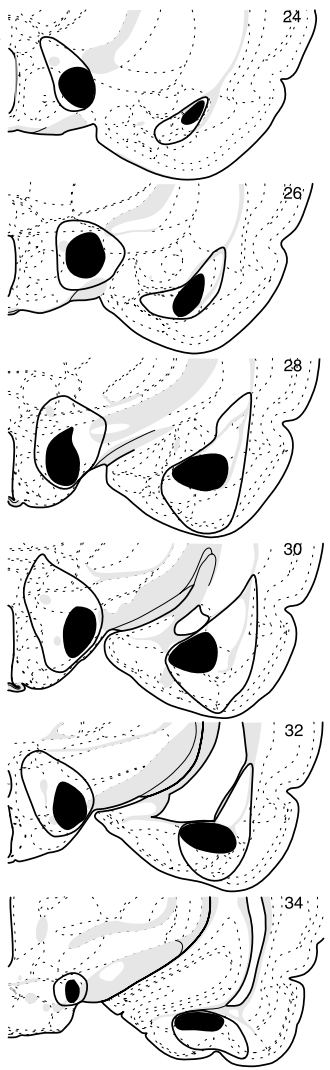

B

LHA sham

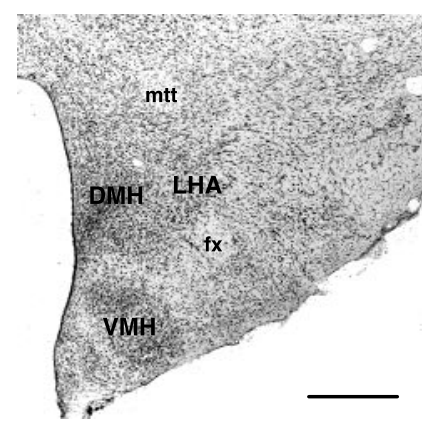

LHA lesion

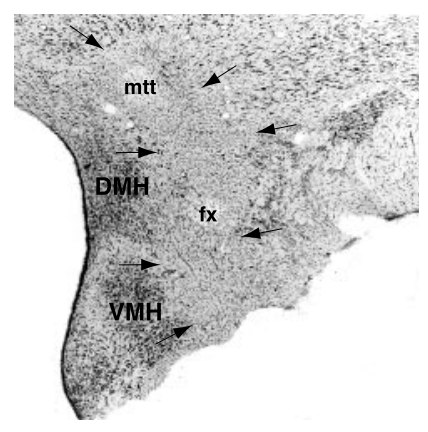

BLA sham

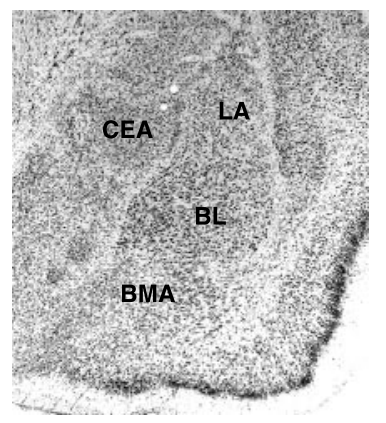

BLA lesion

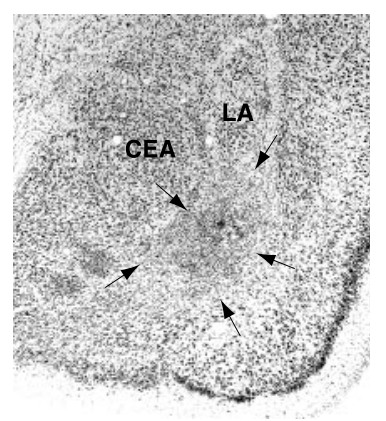

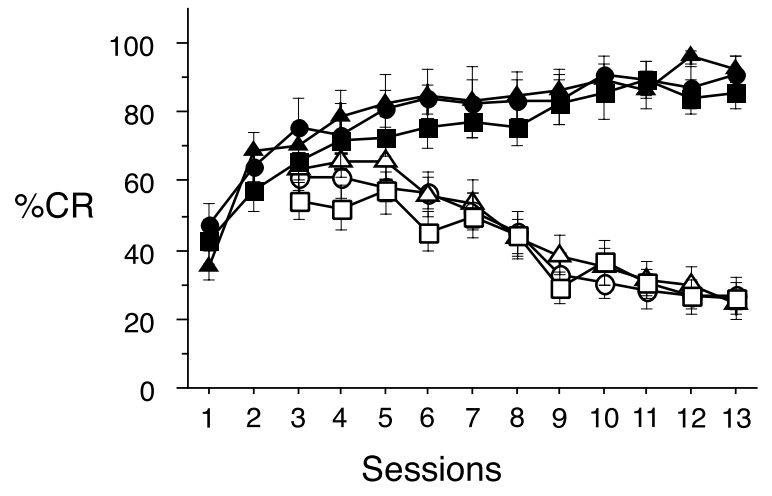

Figure 2. First-order conditioning. Acquisition of discrimination between the CS + and CS - auditory stimuli during the initial phase of training in rats with contralateral $(\mathbf{\square})$, ipsilateral $(\mathbf{\Delta})$, or sham $(\mathbf{O})$ lesions of the BLA and LHA. Conditioned responses to the CS+ are represented by filled symbols, and conditioned responses to the CS- are represented by open symbols. Conditioned responses are expressed as the mean \pm SEM percentage of time expressing food cup behavior.

either the CS+ or the CS- (Fig. 3A). As expected, rats in the sham control group, as well as rats in the ipsilateral lesion group, ate significantly more food in the presence of CS+ compared with CS - (Wilcoxon signed rank; sham group, $p=0.006$; ipsilateral group, $p=0.003)$. In contrast, rats with contralateral lesions ate the same amount of food in both tests $(p=0.3452)$. Analysis of difference scores for consumption during the tests with $\mathrm{CS}+$ and consumption during the tests with $\mathrm{CS}-$ (Fig. 3B) revealed a significant difference among the groups $\left(H_{(2)}=13.968\right.$; $p=0.0009$ ), and subsequent independent Mann-Whitney $U$ tests showed that the sham and ipsilateral groups each differed significantly from the contralateral group $(U=39.0, p=0.004 ; U=$
$14.5, p=0.0004$, respectively), whereas the sham and ipsilateral groups did not differ from one another $(U=90.0 ; p=0.9999)$. The potentiation effect on eating was a result of the previously learned relationship between the cue and food rather than some nonspecific activation by an auditory stimulus, because only the cue paired with food ( $\mathrm{CS}+$ ), but not the explicitly unpaired cue $(\mathrm{CS}-)$ potentiated eating in control groups.

It is also important to note that we did not find any difference in food consumption among the groups $\left(H_{(2)}=0.460 ; p=0.7946\right)$ during a pretesting interval in the absence of either the $\mathrm{CS}+$ or the CS- (baseline condition). Moreover, animals gained the same amount of body weight when food was available ad libitum during the week before tests for potentiated feeding $\left(H_{(2)}=\right.$ 0.496, $p=0.7802$; sham, $71.1 \pm 4.2 \mathrm{gm}$; ipsilateral, $70.9 \pm 4.9 \mathrm{gm}$; and contralateral, $71.7 \pm 4.3 \mathrm{gm})$.

\section{Behavior: second-order conditioning}

Our results demonstrate that BLA and LHA are critical components of a system through which learned cues override satiety and increase eating. It is not certain, however, that direct projections from BLA to LHA are used for this function. Indeed, BLA has a major projection to the nucleus accumbens (ACB), which in turn innervates LHA (Kirouac and Ganguly, 1995). Moreover, ACB-LHA circuitry has been implicated in feeding behavior (Kelley, 1999). In the current study, we tested the same groups of rats in a procedure that is known to depend on the BLA-ACB projection, in which a CS paired previously with food delivery $(\mathrm{CS}+)$ serves as a reinforcer for new learning in second-order conditioning (Setlow et al., 2002). To assess this function, we paired a new stimulus (a light, referred to as CS2) with the original auditory first-order stimulus $(\mathrm{CS}+)$ in additional training sessions after the rats were returned to a food-restricted regimen. Control groups received unpaired presentations of CS2 and 

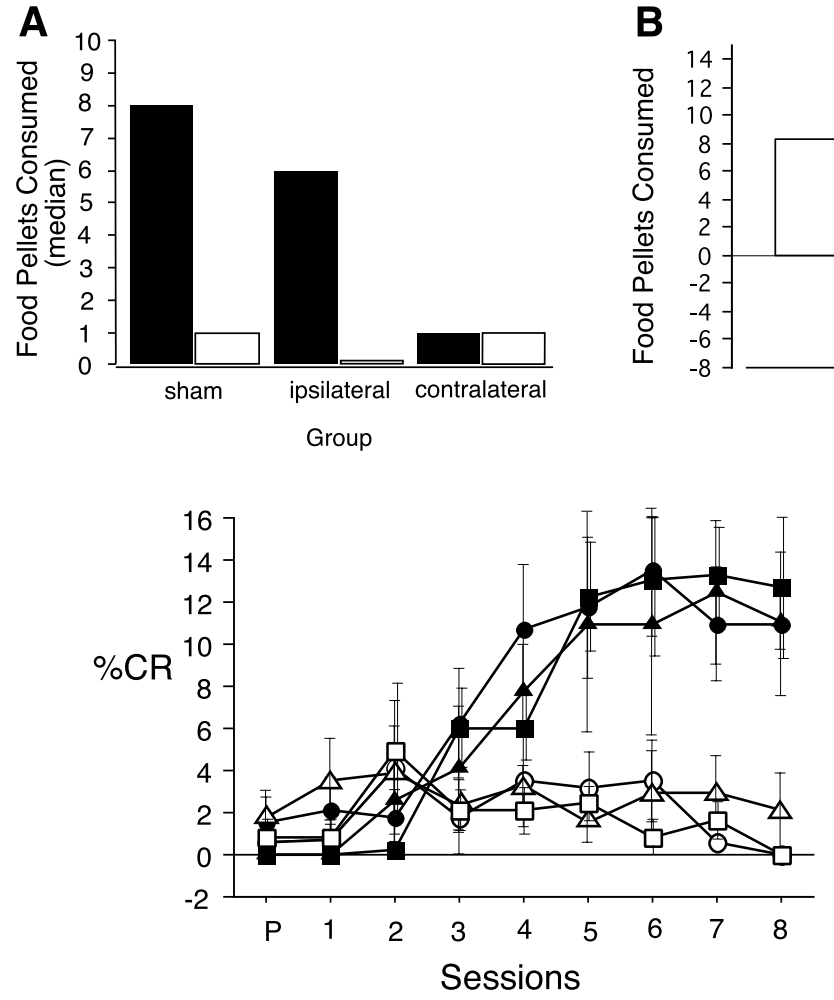

Figure 4. Second-order conditioning. Acquisition of second-order conditioned responses (food cup behavior) to the light CS2 during the second phase of training in rats with contralateral $(\mathbf{\square})$, ipsilateral $(\mathbf{\Delta})$, or sham (๑) lesions of the BLA and LHA. Rats that received paired CS2-CS+ presentations are represented by filled symbols, and rats that received unpaired presentations of CS2 and CS + are represented by open symbols. Session $P$ refers to the pretest of the light at the beginning of training. Conditioned responses are expressed as the mean \pm SEM percentage of time expressing food cup behavior.

$\mathrm{CS}+$. All groups trained with paired presentations acquired comparable conditioned responses to the light CS2, as shown in Figure 4. The difference in conditioned responses to the light between groups given paired, compared with groups given unpaired, presentations of CS2 and CS + was statistically significant (sham, $U=3.500, p=0.0046$; ipsilateral, $U=2.500, p=0.0298$; and contralateral, $U=0.500, p=0.0043$ ). The lesion treatments (sham, ipsilateral, or contralateral) did not produce a significant difference in conditioned responses in either the paired $\left(H_{(2)}=\right.$ $0.088 ; p=0.9570)$ or unpaired $\left(H_{(2)}=1.029 ; p=0.5979\right)$ conditions. In contrast with potentiated feeding, in which a CS augments food consumption, disruption of the BLA-LHA system does not interfere with the ability of a CS to reinforce new learning, indicating some independence of potentiated feeding from other behavioral functions that depend on BLA-ACB projections. It has yet to be determined, however, whether indirect projections from BLA via ACB contribute to potentiated eating.

\section{DISCUSSION}

The present study demonstrated that the BLA-LHA system is crucial for allowing learned cues to override satiety signals and stimulate eating in sated states. This was attributable to an associative process in that eating was augmented by a cue paired previously with food $(\mathrm{CS}+)$ but not an unpaired cue $(\mathrm{CS}-)$. We further showed that the BLA-LHA system is specifically impor- tant for the control of eating by learned signals, because lesions did not affect eating in the pretest baseline sessions or the rate at which rats gained weight when fed ad libitum. As such, this model provides a setting that is highly amenable to a neural systems analysis of learning mechanisms that is uncomplicated by generalized changes in behavioral performance (Cahill et al., 1999; Fanselow and LeDoux, 1999).

If direct projections from the amygdala complex to the hypothalamus mediate the effects of learning on food consumption, that output is likely to arise from the BLA. Although other forms of associative learning, such as fear conditioning, are mediated via projections from the amygdala central nucleus, which receives a large input from the BLA (LeDoux, 2000), this pathway does not appear to be engaged in potentiated feeding. Unlike rats with damage to the BLA, rats with the selective bilateral neurotoxic lesions of the central nucleus exhibit robust potentiated feeding by a conditioned stimulus (Gallagher, 2000; Holland et al., 2002).

A substantial output from the BLA, which originates primarily in the basolateral nucleus, directly innervates the LHA (Petrovich et al., 2001). The exact neurotransmitter used by these projection neurons is not known, although it is likely to be glutamate (Swanson and Petrovich, 1998). In that context, glutamatergic mechanisms within the LHA have been shown to promote feeding in sated rats (Duva et al., 2001). Thus, it is plausible that mechanisms mediating potentiation of feeding, at least in part, involve direct glutamatergic projections from the BLA to LHA.

The exact LHA neurons innervated by the BLA inputs are not known at this time. Nevertheless, it is tempting to speculate that BLA outputs could influence LHA subsystems important for initiation of feeding. For example, groups of LHA neurons express two recently discovered neuropeptides, melanin concentrating hormone and orexin, which are regulated by hunger-satiety state and are linked to initiation of feeding (for review, see Elmquist et al., 1999). Clearly, future work is needed to illuminate the exact neurochemistry of BLA-LHA circuitry and mechanisms mediating control of feeding by learned cues.

In addition to the projections to the LHA, the BLA outputs could reach other parts of the feeding circuit as demonstrated recently with viral labeling techniques (DeFalco et al., 2001). Pseudorabies viruses specifically constructed to infect either neuropeptide Y (NPY)-expressing neurons or neurons expressing the leptin receptor were reported to label BLA neurons after injection into arcuate hypothalamic neurons. The time course for appearance of the BLA labeling suggested transynaptic transport via the LHA and/or ventromedial hypothalamic nucleus. Interestingly, these key feeding-regulatory molecules, leptin and NPY, exert opposite influences on eating. Leptin is linked to 
inhibition of eating (Friedman and Halaas, 1998; Schwartz et al., 2000), whereas NPY is implicated in the initiation of feeding (Leibowitz, 1994; Schwartz et al., 2000), including the induction of eating in food-satiated rats (Stanley et al., 1993). Thus, the BLA could mediate potentiation of eating by learned cues through activation of NPY, inhibition of leptin-mediated mechanisms, or regulation of both of these components of feeding systems.

At the same time, it should be noted that our results do not provide a precise map of how information from the BLA reaches the LHA to modulate feeding in the potentiation paradigm. In addition to direct innervation of the LHA, as mentioned above, the BLA has a major projection to the $\mathrm{ACB}$, which plays a role in feeding behavior at least in part via its connections with the LHA (Kelley, 1999). However, results from the current study indicate some independence in the role of the BLA-LHA and BLA-ACB systems in different behavioral functions that depend on acquired CS value. An intact BLA-LHA system is critical for CS potentiation of feeding but is not necessary for second-order conditioning, which depends on intact BLA-ACB connections (Setlow et al., 2002). Other components of a neural system that modulates feeding on the basis of learning could include indirect innervation of LHA via BLA projections to brain regions that, in turn, project to LHA, including the ventromedial hypothalamus, the bed nuclei of the stria terminalis, substantia innominata, prefrontal cortical areas, or hippocampal formation (Krettek and Price, 1977, 1978; Kita and Oomura, 1982) (for review, see Swanson and Petrovich, 1998; Petrovich et al., 2001).

In the context of the current findings and a large body of previous work (Davis, 1992; Davis and Shi, 1999; Everitt et al., 1999; Fendt and Fanselow, 1999; Holland and Gallagher, 1999; LeDoux, 2000; Maren, 2001), it is clear that the amygdala is key for learning processes whereby neutral stimuli acquire biological significance, as first proposed by Weiskrantz (1956). Additionally, amygdala connections with the hypothalamus have long been thought to play a role in modulation of species-typical behaviors. Referring to the functional neuroanatomy of amydalo-hypothalamic circuits, Kaada (1972) noted that "the amygdala adds plasticity to the basic inborn and more fixed reflex mechanisms."

The present results provide evidence that the amygdalohypothalamic system mediates learned motivational control of feeding. Experience and learning can serve an adaptive function in ingestive behavior, ranging from modifications in an organism's ingestion of particular food items, such as acquired taste aversions or taste preferences, to adaptations in how organisms use information to guide complex behavioral repertoires, such as foraging strategies. In the conditioned potentiation paradigm, cues associated with hunger acquire motivational properties to promote eating even in sated states. Given that food scarcity can occur in natural settings, cues associated with hunger in the past could serve an adaptive function by increasing the tendency to eat.

In addition to BLA inputs that access feeding circuitry in the hypothalamus, the BLA also provides innervation of topographically distinct regions of the hypothalamus implicated in the regulation of other species-typical functions, such as reproductive and defensive behaviors (Petrovich et al., 2001). These projections from the amygdala may also play a key role in mediating adaptive effects of learning that can modulate a range of functions that are basic to survival.

The function of the BLA in the regulation of eating may also have maladaptive consequences. Learned cues that promote eating in food-sated subjects are of special relevance to natural conditions that may induce overeating. Studies in both laboratory animals and humans show that external cues associated previously with food (learned cues) exert powerful control over feeding behavior. Learned cues can override regulatory signals linked to energy balance (Weingarten, 1983; De Castro, 1997), and disturbance in the extrinsic control of feeding systems may cause overeating (Rodin, 1981; Wardle, 1988; Booth, 1989). The present findings have defined components of brain circuitry that allow food-associated cues to override "satiety" signals. This definition is a necessary first step toward development of an animal model in which the external control of feeding behavior and its associated disorders can be better studied.

\section{REFERENCES}

Booth DA (1989) Mood- and nutrient-conditioned appetites. Cultural and physiological bases for eating disorders. Ann NY Acad Sci 575:122-135.

Cahill L, Weinberger NM, Roozendaal B, McGaugh JL (1999) Is the amygdala a locus of "conditioned fear"? Some questions and caveats. Neuron 23:227-228.

Davis M (1992) The role of the amygdala in fear and anxiety. Annu Rev Neurosci 15:353-375.

Davis M, Shi C (1999) The extended amygdala: are the central nucleus of the amygdala and the bed nucleus of the stria terminalis differentially involved in fear versus anxiety? Ann NY Acad Sci 877:281-291.

De Castro JM (1997) Socio-cultural determinants of meal size and frequency. Br J Nutr Suppl 1:S39-S54.

DeFalco J, Tomishima M, Liu H, Zhao C, Cai X, Marth JD, Enquist L, Friedman JM (2001) Virus-assisted mapping of neural inputs to a feeding center in the hypothalamus. Science 291:2608-2613.

Duva MA, Tomkins EM, Moranda LM, Kaplan R, Sukhaseum A, Jimenez A, Stanley BG (2001) Reverse microdialysis of $N$-methyl-Daspartic acid into the lateral hypothalamus of rats: effects on feeding and other behaviors. Brain Res 921:122-132.

Elmquist JK, Elias CF, Saper CB (1999) From lesions to leptin: hypothalamic control of food intake and body weight. Neuron 22:221-232.

Everitt BJ, Parkinson JA, Olmstead MC, Arroyo M, Robledo P, Robbins TW (1999) Associative processes in addiction and reward: the role of amygdala-ventral striatal subsystems. Ann NY Acad Sci 877:412-438.

Fanselow MS, LeDoux JE (1999) Why we think plasticity underlying pavlovian fear conditioning occurs in the basolateral amygdala. Neuron 23:229-232.

Fendt M, Fanselow MS (1999) The neuroanatomical and neurochemical basis of conditioned fear. Neurosci Biobehav Rev 23:743-760.

Friedman JM, Halaas JL (1998) Leptin and the regulation of body weight in mammals. Nature 395:763-770.

Gallagher M (2000) The amygdala and associative learning. In: The amygdala: a functional analysis (Aggleton JP, ed), pp 311-329. New York: Wiley.

Holland P, Gallagher M (1999) Amygdala circuitry in attentional and representational processes. Trends Cogn Sci 3:65-73.

Holland PC, Petrovich GD, Gallagher M (2002) The effects of amygdala lesions on conditioned stimulus-potentiated eating in rats. Physiol Behav 76:117-129.

Kaada MD (1972) Stimulation and regional ablation of the amygdaloid complex with reference to functional representations. In: The neurobiology of the amygdala (Eleftheriou BE, ed), pp 205-281. New York: Plenum.

Kelley AE (1999) Functional specificity of ventral striatal compartments in appetitive behaviors. Ann NY Acad Sci 877:71-90.

Kirouac GJ, Ganguly PK (1995) Topographical organization in the nucleus accumbens of afferents from the basolateral amygdala and efferents to the lateral hypothalamus. Neuroscience 67:625-630.

Kita H, Oomura Y (1982) An HRP study of the afferent connections to rat lateral hypothalamic region. Brain Res Bull 8:63-71.

Krettek JE, Price JL (1977) Projections from the amygdaloid complex to the cerebral cortex and thalamus in the rat and cat. J Comp Neurol 172:687-722.

Krettek JE, Price JL (1978) Amygdaloid projections to subcortical structures within the basal forebrain and brainstem in the rat and cat J Comp Neurol 178:225-254.

LeDoux JE (2000) Emotion circuits in the brain. Annu Rev Neurosci 23:155-184.

Leibowitz SF (1994) Specificity of hypothalamic peptides in the control of behavioral and physiological processes. Ann NY Acad Sci 739:12-35.

Maren S (2001) Neurobiology of Pavlovian fear conditioning. Annu Rev Neurosci 24:897-931. 
McDonald AJ, Culberson JL (1986) Efferent projections of the basolateral amygdala in the opossum, Didelphis virginiana. Brain Res Bull 17:335-350.

Petrovich GD, Canteras NS, Swanson LW (2001) Combinatorial amygdalar inputs to hippocampal domains and hypothalamic behavior systems. Brain Res Rev 38:247-289.

Rodin J (1981) Current status of the internal-external hypothesis for obesity. What went wrong? Am Psychol 36:361-372.

Schwartz MW, Woods SC, Porte Jr D, Seeley RJ, Baskin DG (2000) Central nervous system control of food intake. Nature 404:661-671.

Setlow B, Holland PC, Gallagher M (2002) Disconnection of the basolateral amygdala complex and nucleus accumbens impairs appetitive Pavlovian second-order conditioned responses. Behav Neurosci 116:267-275

Stanley BG, Magdalin W, Seirafi A, Thomas WJ, Leibowitz SF (1993) The perifornical area: the major focus of (a) patchy distributed hypo- thalamic neuropeptide Y-sensitive feeding system(s). Brain Res 604:304-317.

Swanson LW (1992) Brain maps: structure of the rat brain. Amsterdam: Elsevier.

Swanson LW (2000) Cerebral hemisphere regulation of motivated behavior. Brain Res 886:113-164.

Swanson LW, Petrovich GD (1998) What is the amygdala? Trends Neurosci 21:323-331.

United States Department of Health and Human Servivces (1999) National Health and Nutrition Examination Survey, National Center for Disease Control and Prevention.

Wardle J (1988) Cognitive control of eating. J Psychosom Res 32:607-612.

Weingarten HP (1983) Conditioned cues elicit feeding in sated rats: a role for learning in meal initiation. Science 220:431-433.

Weiskrantz L (1956) Behavioral changes associated with the ablation of the amygdaloid complex in monkeys. J Comp Physiol Psychol 49:381-391. 